Theoret. Comput. Fluid Dynamics (1991) 2: 279-290

Theoretical and Computational Fluid Dynamics

(C) Springer-Verlag 1991

\title{
Subgrid Length-Scales for Large-Eddy Simulation of Stratified Turbulence ${ }^{1,2}$
}

\author{
Ulrich Schumann \\ DLR, Institute of Atmospheric Physics, \\ W-8031 Oberpfaffenhofen, Federal Republic of Germany
}

Received 12 November 1990 and accepted 15 March 1991

\begin{abstract}
The influence of buoyancy on the length-scales for the dissipation rate of kinetic energy, and for momentum, heat, and other scalar transport has to be known for subgrid-scale (SGS) models in a large-eddy simulation (LES). For the inertial subrange, Lilly (1967) has shown that grid spacing is the relevant length-scale for SGS effects. Deardorff (1980) proposed to reduce all the length-scales for stable stratification. Numerical and experimental data show, however, that the dissipation length-scale may strongly increase in stable layers with little shear. Lumley's (1964) theory for the energy spectrum in a stratified fluid also suggests such an increase. In this paper we apply the analysis of previous algebraic second-order closure SGS models, parameter studies with different length-scale models in LES, and the analysis of direct simulations of sheared and unsheared stably stratified homogeneous turbulence. These analyses show advantages of firstorder closures for LES and suggest that the limiting effect of stratification should only be applied to the length-scales of vertical eddy-diffusivities of heat and scalars but not to those of momentum and dissipation.
\end{abstract}

\section{Introduction}

A large-eddy simulation (LES) resolves the energy-carrying motions but requires models for the mechanical dissipation rate and turbulent diffusivities by subgrid-scale (SGS) motions. If the grid scale $\Delta$ is small enough so that the subgrid turbulence behaves as locally isotropic and follows the Kolmogorov energy spectrum, then $\Delta$ and the SGS kinetic energy $e$ are the controlling scales for the SGS model. In this case, the following models for the dissipation rate $\varepsilon$ and for the turbulent diffusivities $K_{\mathrm{m}}, K_{\mathrm{h}}$, and $K_{\mathrm{c}}$, for momentum, heat, and scalar concentrations, respectively,

$$
\varepsilon=\frac{e^{3 / 2}}{l_{\varepsilon}}, \quad K_{\mathrm{m}}=l_{\mathrm{m}} e^{1 / 2}, \quad K_{\mathrm{h}}=K_{\mathrm{c}}=l_{\mathrm{h}} e^{1 / 2}, \quad l_{\varepsilon}=\frac{l}{c_{\varepsilon}}, \quad l_{\mathrm{m}}=c_{\mathrm{m}} l, \quad l_{\mathrm{h}}=c_{\mathrm{h}} l,
$$

can be deduced as shown by Lilly (1967) (details as in Schmidt and Schumann, 1989) with

$$
\begin{gathered}
l=\Delta, \quad c_{\varepsilon}=\left(\frac{2}{3 \alpha}\right)^{3 / 2} \pi=0.845 \\
c_{\mathrm{m}}=\left(\frac{2}{3 \alpha}\right)^{3 / 2} \frac{1}{\pi}=0.0856, \quad c_{\mathrm{h}}=\left(\frac{2}{3 \alpha}\right)^{1 / 2} \frac{4}{3 \gamma} \frac{1}{\pi}=0.204,
\end{gathered}
$$

\footnotetext{
${ }^{1}$ Dedicated to Professor J.L. Lumley on the occasion of his 60th birthday.

${ }^{2}$ This work was supported by the Deutsche Forschungsgemeinschaft.
} 
where $\alpha=1.6$ and $\gamma=1.34$ are the Kolmogorov and Batchelor coefficients, i.e., the coefficients in the spectra of kinetic energy and temperature variance in the inertial subrange. In order to apply this model, a budget equation for the SGS energy $e$ has to be integrated which includes shear production, buoyancy production, dissipation, diffusion, and advection of kinetic energy (see the Appendix). In contrast to second-order closure (SOC) models (Deardorff, 1973; Launder, 1975; Mellor and Yamada, 1982), which solve additional budget equations for the fluxes, we call this a first-order closure model, although it contains the SOC's energy equation. In the classical Smagorinsky-Lilly model, see Lilly (1967), as used by Mason and Derbyshire (1990), the eddy diffusivities are proportional to the magnitude of local (resolvable) velocity deformation and to the square of the grid interval together with corrections as a function of the Richardson number. Schmidt and Schumann (1989) followed earlier proposals (e.g., Schemm and Lipps, 1976), and used an algebraically approximated SOC model. This model works well for small deviations from locally isotropic SGS turbulence, but the model is rather complex and has some realizability problems, as is explained in the Appendix. Moreover, as we show in this paper, the advantages of this model are small in comparison with a first-order closure model. For these reasons, we now (Schumann, 1990; Krettenauer, 1991) prefer to use the first-order closure, i.e., (1) and (2).

For stably stratified turbulence, the small-scale motions are no longer isotropic and vertical diffusion and dissipation are reduced. Deardorff (1973) found that the model given in (1) and (2) overestimates vertical SGS fluxes in stably stratified layers above the atmospheric boundary layer. Therefore, similar to Brost and Wyngaard (1978), Deardorff (1980) proposed reducing the length-scale $l$ to a buoyancy scale $l_{\mathrm{b}}$ which describes the height to which a fluid parcel of a given kinetic energy can rise until all the kinetic energy is converted into potential energy,

$$
l=\min \left(\Delta, l_{\mathrm{b}}\right), \quad l_{\mathrm{b}}=\frac{0.76 e^{1 / 2}}{N},
$$

as a function of SGS kinetic energy $e$ and the local Brunt-Väisälä frequency $N$ of the stratified fluid. The coefficient 0.76 has no formal justification. For isotropic turbulence the coefficient should be $\left(\frac{2}{3}\right)^{1 / 2}=0.82$ instead; for realistic anisotropy, the coefficient could be much smaller. The buoyancy scale $l_{\mathrm{b}}$ may become very small and this may cause numerical problems, in particular in the integration of the budget equation of SGS kinetic energy in which $l$ enters inversely in the dissipation rate.

A reduction of diffusion-related length-scales by stable stratification appears physically reasonable in view of the limited vertical displacements which fluid parcels can undergo in a stably stratified fluid layer. However, it is not clear whether the dissipation length-scale should be reduced in stably stratified flows because such flows may exhibit large kinetic energy from wavy motions with little energy transfer toward the smaller dissipating scales. In fact, Lumley's (1964) result for the energy spectrum in stratified turbulence as a function of wave number $k$,

$$
\hat{E}(k)=\alpha \varepsilon^{2 / 3}\left\{1+\left(\frac{k}{k_{\mathrm{b}}}\right)^{-4 / 3}\right\} k^{-5 / 3}, \quad k_{\mathrm{b}} \cong \alpha^{-3 / 4}\left(\frac{N^{3}}{\varepsilon}\right)^{1 / 2}
$$

integrated from $k_{0}=2 \pi / \Delta$ to infinity, for $k_{0}<k_{\mathrm{b}}$,

$$
e \cong\left(\frac{\pi^{2}}{8}\right) \Delta^{2} N^{2}+\frac{\frac{1}{2} \alpha^{3 / 2} \varepsilon}{N},
$$

shows a first part of SGS energy that is related to wavy motions which are damped by buoyancy forces, while only the second part measures inertial-range energy. The inverse of $k_{\mathrm{b}}$ approximately equals the Ozmidov-scale (Ozmidov, 1965); this scale has to be much larger than $L_{\mathrm{K}}=\left(v^{3} / \varepsilon\right)^{1 / 4}$, the Kolmogorov scale, in order to have overturning (active) turbulence (Vinnichenko et al., 1973; Gerz and Schumann, 1991). Lumley's spectrum predicts a $k^{-3}$-shape for $k \ll k_{\mathrm{b}}$. Such spectral shapes are observed in the stratosphere, but, as discussed by Weinstock (1990), possibly for other reasons (breaking of saturated gravity waves instead of local shear and buoyancy interaction).

Schmidt and Schumann (1989) neglected the effect of stratification on $l$ but nevertheless found very good agreement with available data for the case of the atmospheric and the laboratory convective 


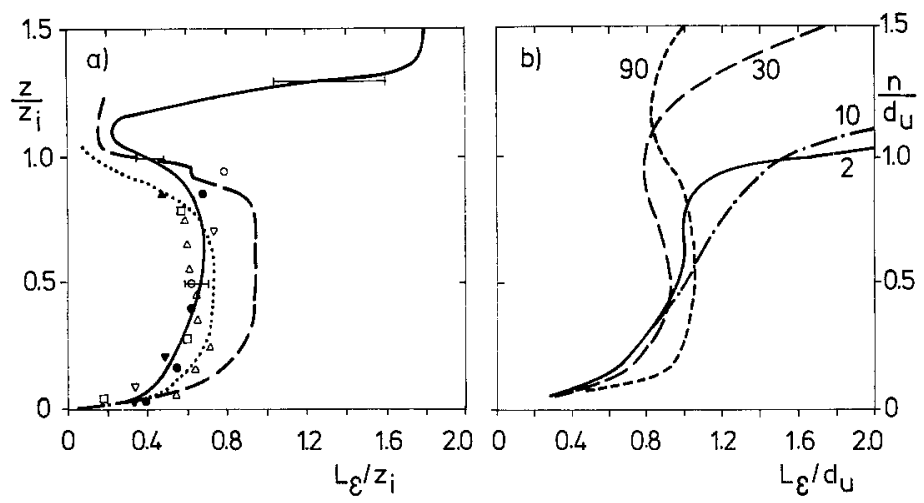

Figure 1. (a) LES results of $L_{\varepsilon}=E^{3 / 2} / \varepsilon$ versus height $z$ for the CBL normalized by its height $z_{i}$ as obtained by Schmidt and Schumann (1989) (full curve with error bars), by Moeng and Wyngaard (1989) (dashed curve), by the model of Bougeault and Lacarrère (1989) (dotted), and from measurements in the atmosphere and the laboratory (symbols taken from Bougeault and Lacarrère, 1989). (b) Same for the upslope boundary layer for various slope angles $\alpha\left(\alpha=90^{\circ}\right.$ for a vertical wall) from Schumann (1990). The vertical coordinate denotes the normal distance $n$ above a sloping surface normalized by the thickness $d_{u}$ of the upsiope layer which is the lateral position of the first zero of the upslope velocity.

boundary layer (CBL) which is topped by a stable inversion and weakly stable fluid above the inversion. In fact their results are very similar to those obtained by Mason (1989), Moeng and Wyngaard (1989), and Nieuwstadt (1990), who used Deardorff's limitation by $l_{\mathrm{b}}$.However, Ebert et al. (1989), who simulated the dispersion of passive tracers using the LES method of Schmidt and Schumann (1989), found rather large dispersion of tracers above the inversion which suggests that $l$ should be reduced in the stable layer.

From LES, we may deduce statistics of all turbulent scales. For example, Moeng and Wyngaard (1989) computed the length-scale $L_{\varepsilon}=E^{3 / 2} / \varepsilon$, which is related to the kinetic energy $E=\overline{u_{i}^{\prime 2}} / 2$ (we apply the summation convention) of all motion scales (actually they evaluated $L_{2}=2^{3 / 2} L_{\varepsilon}$ and determined $\varepsilon$ from the residuum of the energy balance whereas we determine $\varepsilon$ from the SGS model). The length-scale $L_{\varepsilon}$ is of utmost importance in SOC models which use prescribed length-scales as in Mellor and Yamada (1982) and others. Moeng and Wyngaard (1989) found that $L_{\varepsilon}$ is much larger than the predictions of previous SOC models within the mixed layer of the CBL. Figure 1(a) plots the result which we deduced from their data for both the mixed layer and the stable layer above the inversion. Also plotted are the results which follow from the LES of Schmidt and Schumann (1989). We observe that both models give about the same result for the mixed layer but $L_{\varepsilon}$ is much larger from the latter study above the inversion. This increase of $L_{\varepsilon}$ contradicts the expectation which forms the basis of Deardorff's (1980) proposal. Figure 1(a) also includes data points which Bougeault and Lacarrère (1989) deduced from various atmospheric and laboratory measurements and results from their parametrization. The data generally confirm our LES results. The parametrization also agrees very well with the LES results but predicts smaller $L_{\varepsilon}$ near and above the inversion. Druilhet et al. (1983) and Isaka and Guillemet (1983) measured dissipation and variances of vertical velocity in and above atmospheric CBLs. Unfortunately, they did not measure the horizontal velocity variance so we cannot compute $E$. But even if we assume zero horizontal variance, their measurements imply an exponential increase in $L_{\varepsilon}$ above the inversion.

Figure 1(b) replots the result which was obtained in Schumann (1990) from LES of the upslope boundary layer for various slope angles. As shown in Schumann (1990), turbulence in the slope layer is driven mainly by buoyancy at small angles but mainly by shear at large angles. We note that $L_{\varepsilon}$ outside the boundary layer decreases with increasing slope angle. This suggests that the length-scale decreases with increasing importance of shear and becomes large for unsheared stable layers.

Hunt et al. $(1988,1989)$ show that for $R i$ less than half, it is the mean shear $S=d U / d z$ which determines $L_{\varepsilon}$. They propose $L_{\varepsilon}^{-1}=A_{\mathrm{B}} / z+A_{\mathrm{s}} S / w^{\prime}+1 / L_{0}$. Here, $w^{\prime}$ is the rms-value of vertical velocity fluctuations, $L_{0}$ is the overall scale of the flow, and $A_{\mathrm{B}} \cong 0.17$ and $A_{\mathrm{s}} \cong 0.46$ are coefficients determining the relative importance of "blocking" by the lower surface and shear effects. Mason and Derbyshire (1990) tested Hunt's model and that of Brost and Wyngaard (1978) by comparing LES 
results on dissipation with the model's prediction in neutral and stable layers, and found that Hunt's model compares better.

As discussed by Bougeault and Lacarrère (1989), Lilly et al. (1974), Weinstock (1978), and others deduced

$$
K_{\mathrm{h}}=C_{\mathrm{h}} \varepsilon N^{-2}
$$

with various values of $C_{\mathrm{h}} \cong 0.81$ for the vertical turbulent diffusion coefficient in the stratosphere. Weinstock (1990) has recently questioned this result; he now "believes" $C_{\mathrm{b}}$ to be close to $\frac{1}{4}$ (it might be noted that Weinstock uses a theoretical model with $K_{\mathrm{m}}=K_{\mathrm{h}}$, i.e., a constant turbulent Prandtl number). Hunt et al. (1985) relate the diffusivity of heat to the variance of vertical velocity fluctuations,

$$
K_{\mathrm{h}}=\frac{C_{f} \overline{w^{\prime 2}}}{N} \quad \text { with } \quad C_{f}=0.17 \pm 0.08
$$

Mason and Derbyshire (1990) show that $C_{f}$ is actually a function of the Richardson number, at least for Richardson numbers less than the critical one.

Zeman and Lumley (1976), Launder (1989), and others developed SOC models including budget equations for the dissipation rate $\varepsilon$. They did not explicitly evaluate the magnitude of $L_{\varepsilon}$.

Gerz and Schumann (1991) studied homogeneous stratified turbulence with and without shear by direct simulations (without SGS modeling) for moderate Reynolds numbers $\left(R e_{\lambda}=26.4\right)$. They found that shear increases dissipation for stably stratified flows but did not compute the value of the dissipation length-scales. As these simulations do not resolve the inertial range of turbulence, such an analysis can be of qualitative value only.

In this paper we investigate the following questions:

1. What consequences result from the energy balance, realizability, and the SOC model with respect to the model for SGS length-scales?

2. How sensitive are LESs of turbulence and scalar dispersion with respect to the assumed model for these scales?

3. What do the results of direct simulations of stably stratified sheared and unsheared homogeneous turbulence suggest with respect to the scales?

\section{The Energy Budget and Length-Scales}

We consider the budget of SGS kinetic energy $e$ in homogeneous turbulence under shear $S=d U / d z$ (neglecting other shear components for brevity) and stable stratification, $d T / d z>0$,

$$
\frac{d e}{d t}=-\overline{u^{\prime} w^{\prime}} S+\beta g \overline{w^{\prime} T^{\prime}}-\varepsilon
$$

Taking gradient diffusivity approximations to the fluxes and the inertial-range approximation to dissipation,

$$
\overline{u^{\prime} w^{\prime}}=-l_{\mathrm{m}} e^{1 / 2} S, \quad \overline{w^{\prime} T^{\prime}}=-l_{\mathrm{h}} e^{1 / 2} \frac{d T}{d z}, \quad \varepsilon=\frac{e^{3 / 2}}{l_{\varepsilon}},
$$

implies, in steady state, with $N^{2}=\beta g d T / d z$ ( $\beta$ is the volumetric expansion coefficient, $g$ is gravity, and $N$ is the Brunt-Väisälä frequency),

$$
e=l_{\varepsilon}\left(l_{\mathrm{m}} S^{2}-l_{\mathrm{h}} N^{2}\right) .
$$

Realizability (in a much simpler way than discussed in Schumann (1977)) requires that the bracketed expression stays nonnegative for vanishing energy, i.e.,

$$
\frac{l_{\mathrm{m}}}{l_{\mathrm{h}}} \geq R i=\frac{N^{2}}{S^{2}} \quad \text { for } \quad e \rightarrow 0 .
$$

SOC models, as deduced by Mellor and Yamada (1982), show that the vertical heat flux becomes reduced for stable stratification. A similar SOC model has been used in Schmidt and Schumann 
Figure 2. Turbulent Prandtl number $P r_{\mathrm{t}}$ normalized with its value for neutral stratification versus gradient Richardson number $R i$. Dots and circles denote experimental data by Webster (1964), the dashed curve is Launder's (1975) model result, error bars indicate the results from direct numerical simulations of Gerz et al. (1989), and the full curve depicts $P r_{\mathrm{t}} / P r_{\mathrm{to}}=1+0.3 S h^{2} R i$ for $S h=3.01$.

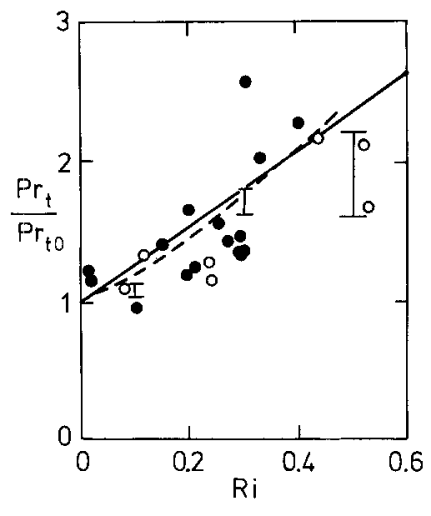

(1989), see the Appendix. In fact, from that model, (41), we find, for strong positive vertical temperature gradients (i.e., for $\left.\left(\partial T / \partial x_{i}\right)^{2} \cong(\partial T / \partial z)^{2}\right)$,

$$
l_{\mathrm{h}}=\frac{c_{\mathrm{h}} l}{1+0.3 l^{2} N^{2} / e}, \quad l_{\mathrm{m}}=c_{\mathrm{m}} l, \quad l_{\varepsilon}=\frac{l}{c_{\varepsilon}},
$$

where $l=\Delta$ is taken as the controlling scale for the SGS motions. This model is consistent with the realizability constraint, since $l_{\mathrm{h}} \rightarrow 0$ for $e \rightarrow 0, N^{2}>0$. Moreover, (12) implies a turbulent Prandtl number

$$
\operatorname{Pr}_{\mathrm{t}}=\frac{K_{\mathrm{m}}}{K_{\mathrm{h}}}=\frac{l_{\mathrm{m}}}{l_{\mathrm{h}}}=\frac{\left(1+0.3 \Delta^{2} N^{2} / e\right) c_{\mathrm{m}}}{c_{\mathrm{h}}} .
$$

The variation of $\operatorname{Pr}_{\mathrm{t}}$ with Richardson number $R i$ agrees perfectly with the results of Gerz et al. (1989), see Figure 2, if we set $\Delta^{2} N^{2} / e=S h^{2} R i$, where $S h=S \Delta / e^{1 / 2}$ is the shear number, and use $S h=3.01$ as in the simulations. As a further point, the above result for $l_{\mathrm{h}}$ implies an eddy diffusivity for heat, $K_{\mathrm{h}}=l_{\mathrm{h}} e^{1 / 2}$, which satisfies

$$
K_{\mathrm{h}}=\frac{c_{\mathrm{h}}}{0.3} \frac{e^{3 / 2}}{\Delta N^{2}}=\frac{c_{\mathrm{h}}}{0.3} \frac{\varepsilon}{c_{\varepsilon} N^{2}},
$$

in the limit to very stable stratification, i.e., for $\Delta^{2} N^{2} / e \gg 1$. This result is consistent with (6), if $C_{\mathbf{h}}=0.794$. The close agreement with the result 0.81 given by Weinstock (1978) should be taken as incidental but shows that our analysis is consistent with previous theories. It is also consistent with Hunt et al. (1985, equation (7)), if $\overline{w^{\prime 2}} / E$ scales as $E /(N \Delta)$, i.e., if the anisotropy of the velocities becomes large for strong stability, which is reasonable. Hence, (12) gives a reasonable set of lengthscales for a first-order SGS model. In an SOC model, the length-scales for momentum and heat should have a constant ratio because the stratification effects enter such a model explicitly.

\section{Sensitivity Study Using LES of the CBL}

We apply the LES method of Schmidt and Schumann (1989) to the buoyancy-driven CBL (with zero mean shear) using either the algebraic SOC model as summarized in the Appendix, or one of the following alternative versions of first-order SGS models:

(A) (Constant SGS.) Equations (1) and (2) with $l=\min \left(\Delta, c_{L} z\right.$ ).

(B) (Deardorff-type model.) Same as (A) but $l=\min \left(\Delta, c_{L} z, l_{\mathrm{b}}\right)$ and $l_{\mathrm{b}}=\max \left(0.1 \Delta, 0.76 e^{1 / 2} / N\right)$. The lower limit $0.1 \Delta$ for $l_{\mathrm{b}}$ has been included heuristically to avoid division by zero.

(C) (Stability limited vertical scalar diffusion.)

$$
\varepsilon=c_{\varepsilon} \frac{e^{3 / 2}}{l}, \quad K_{\mathrm{m}}=c_{\mathrm{m}} l e^{1 / 2}, \quad K_{\mathrm{h}}=K_{\mathrm{c}}=c_{\mathrm{h}} \frac{l e^{1 / 2}}{1+0.3 \Delta^{2} N^{2} / e},
$$

with $l=\min \left(\Delta, c_{L} z\right)$ as in case A. For horizontal fluxes, $K_{\mathrm{h}}=K_{\mathrm{c}}=c_{\mathrm{h}} l e^{1 / 2}$.

In all cases, $c_{L}=0.845$. 

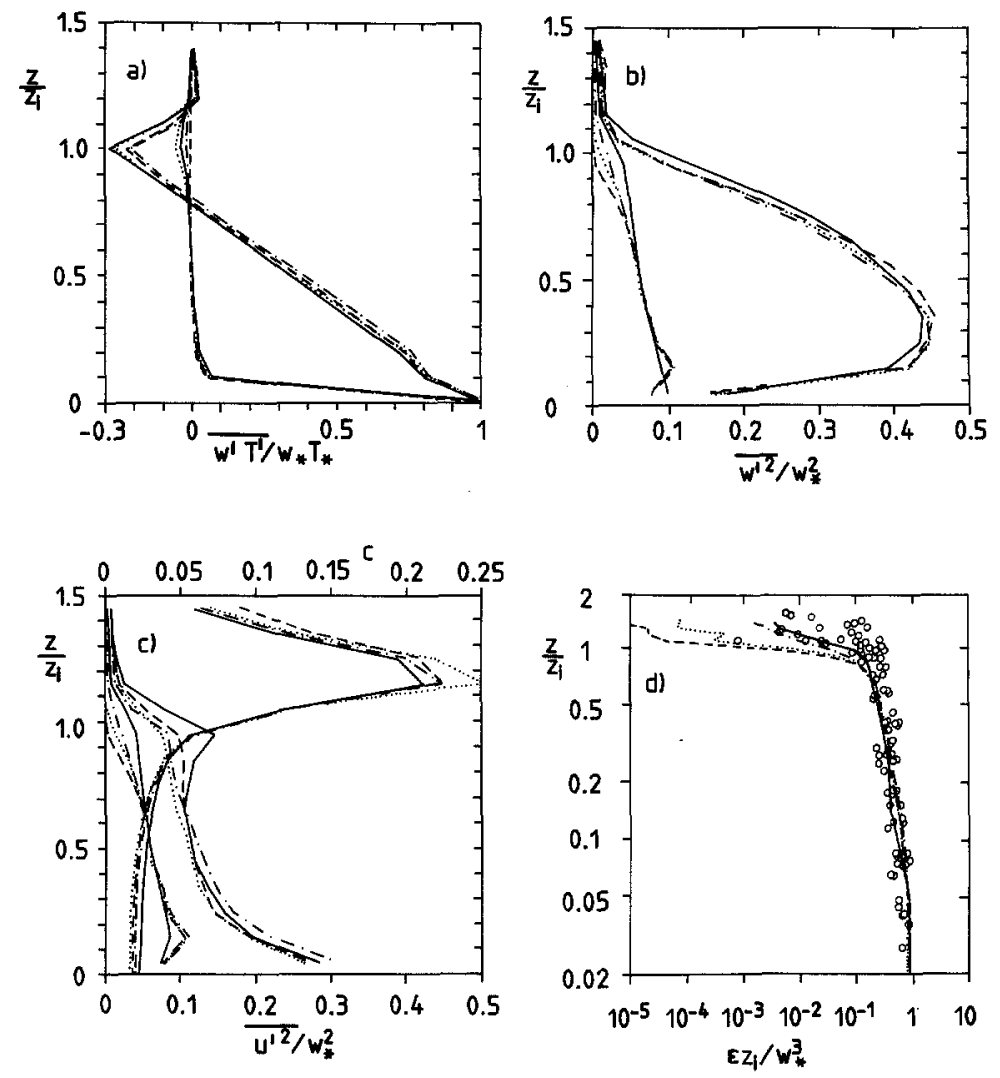

Figure 3. LES results for a CBL at $t=7 z_{i} / w_{*}$ as obtained for the SOC model (full curves) and for the first-order closure models, versions A (dotted), B (dashed), and C (dash-dotted), showing normalized profiles of (a) vertical temperature flux, (b) vertical velocity variance, (c) horizontal velocity variance $\overline{u^{\prime 2}}$ and concentration of a scalar $c$, and (d) dissipation rate in logarithmic scales; the circles represent data from Guillemet et al. (1983) for a CBL with little mean wind. In (a)-(c) total and SGS contributions are plotted separately.

In order to exhibit clearly the effect of the SGS model, we use a rather coarse grid compared with that used in Schmidt and Schumann (1989) $(40 \times 40 \times 15$ instead of $160 \times 160 \times 48)$ and a reduced horizontal domain size ( 3.2 instead of 5 times the height $z_{i}$ of the CBL). The computing time for such a run on a CRAY-YMP amounts to $450 \mathrm{~s}$. All other parameters are as in the previous paper. The results are normalized by the convective scales $w_{*}=\left(\beta g \overline{w^{\prime} T_{\mathrm{s}}^{\prime}} z_{i}\right)^{1 / 3}, T_{*}=\overline{w^{\prime} T_{\mathrm{s}}^{\prime}} / w_{*}$, and $z_{i}$, where $\overline{w^{\prime} T_{\mathrm{s}}^{\prime}}$ is the surface temperature flux.

Figure 3 shows those mean profiles which are most sensitive to the model variants. We see that the mean profiles are generally as those obtained for much finer resolution by Schmidt and Schumann (1989). The SGS contributions are small throughout the mixed layer even for the coarse resolution. The differences between the various model versions are small. Some differences can be noted, however: the SGS heat flux at the inversion $\left(z / z_{i} \cong 1\right)$ becomes negative in version $\mathrm{A}$ but is small or even slightly positive in versions $\mathrm{B}$ and $\mathrm{C}$ which use stability-limited length-scales. In the models this comes from the fact that downward (negative) heat fluxes are related to a very stable stratification for which the smaller scale $l_{\mathrm{b}}$ becomes effective, whereas positive fluxes occur in unstable parts of the flow where the larger scale $\Delta$ applies. Therefore, on average, the SGS flux becomes positive. This is an advantage because spectral analysis of the entrainment heat-flux has shown (see Figure 12 of Schmidt and Schumann, 1989) that the small-scale flux should be positive. In fact, such positive SGS fluxes have been computed by Moeng and Wyngaard (1989) using the stability-limited length-scales whereas the SGS flux in Schmidt and Schumann (1989) was negative at the inversion.

The SGS variances, see Figure 2(b) and (c), near the inversion are largest in the SOC model but smallest in version B. Version C is close to the SOC results. In the lower part of the mixed layer, all model versions give about the same results, except for the lowest two grid levels, where the SOC 
model gives a smoother profile. But the differences in the resolved variances are very small. Hence, changes in the length-scales affect the results significantly only in the stable part.

Important differences can also be seen in Figure 3(d) where we compare the dissipation results with measurements of Guillemet et al. (1983). Below the inversion, data and simulations agree quite well for all model versions. At the inversion and above, however, models $\mathrm{A}$ and $\mathrm{B}$ underestimate the dissipation data considerably, whereas the SOC version and version $\mathrm{C}$ are quite close to the data. Hence, it is essential that the SGS model accounts for the reduced vertical heat flux at the inversion whereas reduction of the dissipation scale, as in version B, gives the wrong trend. Moreover, versions A and B have realizability problems. This can be seen from the small values of dissipation which reflect very small SGS energies. In fact, we had to enforce the energy to stay above a limit, like $10^{-6} w_{*}^{2}$, in versions $\mathrm{A}$ and $\mathrm{B}$, in order to prevent the energy $e$ from becoming negative.

Figure 3(c) shows vertical profiles of tracer concentration $c$ at the final time of the simulations. This tracer was initialized such that it is zero everywhere except in the 12th grid layer $\left(1.1 \leq z / z_{i}<1.2\right)$ where it is set to unity at $t=0$. Because of stable stratification at that level, we expect very little dispersion. The LES results show, however, that the concentration peak became reduced to less than 0.25 . The peak concentrations in the various versions differ by up to $18 \%$ relative to each other. Concentration stays largest in version A, whereas variants B and C, in spite of reduced length-scales, give stronger dispersion. This is surprising and cannot be explained completely. As discussed by Ebert et al. (1989), some of the resultant differences might be caused by numerical diffusion induced by the discrete approximation of advection with the resolved vertical motions. Smaller vertical motions cause less numerical diffusion.

In general, however, version $\mathrm{C}$ is in close agreement with the SOC results. Hence, the simple model $\mathrm{C}$ gives satisfactory results in most respects, even for the coarse grid.

\section{Length-Scales in Sheared and Unsheared Stratified Turbulence}

From direct simulations of Gerz and Schumann (1991), we evaluate SGS energies and dissipation rates

$$
e(\Delta)=\int_{2 \pi / \Lambda}^{\infty} \hat{E}(k) d k, \quad \varepsilon(\Delta)=8 \pi^{2} v \int_{2 \pi / \Lambda}^{\infty} k^{2} \hat{E}(k) d k,
$$

where $v$ is the molecular viscosity, $\hat{E}(k)$ is the actual energy spectrum of the simulated results, and $\Delta$ is the assumed grid scale of an LES representation of the flow. The integrals are actually implemented as sums over the discrete wave-number space where the upper limit ends at the maximum wave number resolved by the $64^{3}$ grid of the direct simulations. From the results we compute the SGS dissipation-scale $l_{\varepsilon}(\Delta)=e(\Delta)^{3 / 2} / \varepsilon(\Delta)$. In Figure $4 l_{\varepsilon} / \Delta$ is plotted for various values of $\Delta / L$ at time $v_{0} t / l_{0}=3$ of the direct simulations, where $v_{0}$ and $l_{0}$ are the rms velocity and the integral length-scale of the turbulent fields at initialization of the direct simulations. Results at nondimensional times 2 and 4 look qualitatively similar. The results are plotted versus the normalized Brunt-Väisälä frequency $N l_{0} / v_{0}$ which is identical to the inverse of the Froude number $F$ for unsheared turbulence and which

Figure 4. Direct numerical simulation results for dissipation length-scale $l_{\varepsilon}=e^{3 / 2} / \varepsilon$, stability-scale $l_{\mathrm{b}}=e^{1 / 2} / N$, and (in the sheared case) the shear limited scale $l_{\mathrm{s}}=\left(\Delta^{-1}+A_{\mathrm{s}} S / e^{1 / 2}\right)^{-1}$, over grid-scale $\Delta$ for (a) unsheared and (b) sheared turbulence versus normalized Brunt-Väisälä frequency $N$ at time $t v_{0} / l_{0}=3$ for various values of $L / \Delta . L=4 \pi, l_{0}=0.9044, v_{0}=0.8226$, and $\operatorname{Re}_{I}=v_{0} l_{0} / v=42.7$.

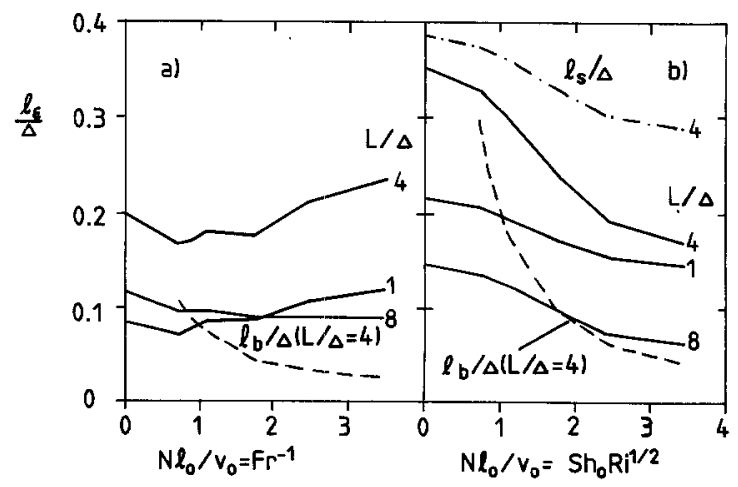


equals the product of the shear number $S h_{0}=S\left(l_{0} / v_{0}\right)$ times the square root of the gradient Richardson number $R i$ in the sheared cases.

From (1) and (2) we expect $l_{\varepsilon} / \Delta=e^{3 / 2} /(\varepsilon \Delta)=c_{\varepsilon}^{-1} \cong 1.18$, for high Reynolds number turbulence with $\Delta$ in the inertial subrange. However, Figure 4 shows that $l_{\varepsilon} / \Delta$ depends on $\Delta$ and is smaller than this value. Obviously, the Reynolds number (as dictated by the limited grid) is far too low to resolve the inertial range. In fact, at the end of the simulation period for $N l_{0} / v_{0} \geq 2$, the direct simulations reach a state where the Ozmidov scale becomes small and comparable with the Kolmogorov scale. Therefore, we must be cautious in conclusions with respect to high Reynolds number flows. Nevertheless, the results show clearly different trends of the dissipation scale with increasing stratification. The scale $l_{\varepsilon}$ increases slightly in the unsheared case but decreases in the sheared case. The changes in the scale are caused by changes in the energy spectrum. Larger scales imply a steeper decrease of the energy spectrum with increasing wave number and vice versa. In the unsheared case, stable stratification suppresses the nonlinear interactions so that less energy is cascading down to small-scale motions. This is corroborated by results on the velocity-derivative skewness shown by Gerz and Schumann (1991) which decreases with increasing stability. On the other hand, shear excites smallscale motions either directly (if there is momentum transfer at small scales) or by re-establishing the cascade process. As discussed by Gerz and Schumann (1991), shear may cause wave-breaking at small scales due to an effectively smaller Richardson number at smaller scales. This interpretation is consistent with results of Thorpe (1978) which show that shear enhances breaking of internal waves. Such wave-breaking enhances small-scale motions with the effect that dissipation grows more (or decreases less) than energy above the cut-off wavelength $\Delta$ with the effect of decreasing length-scale $l_{\varepsilon}$.

These results have consequences with respect to the ratio of the scales for momentum and heat transfer. If $l_{\mathrm{m}}$ would decrease like $l_{\mathrm{h}}$ for strong stratification, then the SGS model would be unable to model the shear effect on stratified flows. Therefore, these results corroborate that $l_{\mathrm{m}}$ should stay finite even for strong stratification because otherwise shear production would become small.

Also plotted in Figure 4 are the stability-scale $l_{\mathrm{b}}=e^{1 / 2} / N$ and the shear-scale $l_{\mathrm{s}}$ as in Hunt et al. (1988, 1989) for $A_{\mathrm{s}}=0.46$. We see that $l_{\mathrm{b}}$ decreases much more quickly than $l_{\varepsilon}$ with increasing stratification and, hence, (3) overestimates the effect of stratification. On the other hand, $l_{\mathrm{s}}$ approximates the trend of $l_{\varepsilon}$ correctly, although some adjustment of the numerical coefficients might be required for an SGS model.

\section{Conclusions and Discussion}

With regard to the questions stated in the introduction we conclude from the results of this study:

1. Our analyses based on the energy budget, its realizability, and on the algebraic SOC model of Schmidt and Schumann (1989) imply SGS length-scales for first-order gradient-diffusion approximation as given in (12) and (15).

2. LESs of turbulence and scalar dispersion are only weakly sensitive with respect to the assumed model for the SGS length-scales. Even for a very coarse grid, the differences induced by various models are less than about $18 \%$ for the maximum concentration results. However, there are some features, like the SGS entrainment flux and dissipation at the inversion and above in the stably stratified parts, which are obviously more correctly represented by a first-order model with a reduced scale for $l_{\mathrm{h}}$ in the stably stratified part.

3. Results of direct simulation of stably stratified sheared and unsheared homogeneous turbulence at moderate Reynolds number show that the SGS dissipation-scale stays about constant or even increases slightly with increasing stratification in an unsheared flow but decreases in sheared cases, at least for the Reynolds numbers the direct simulations can tackle. Even with shear, the decrease is much less than predicted by Deardorff (1980). Therefore we do not recommend his proposal. In strongly sheared turbulence, it might be reasonable to replace $\Delta$ by $\left(\Delta^{-1}+A_{s} S / e^{1 / 2}\right)^{-1}$. The value $A_{s}=0.46$ given by Hunt et al. (1989) cannot be confirmed by direct simulations but may be used as a first guess for SGS modeling.

This dissipation length-scale should also be predictable by SOC models, as those of Zeman and Lumley (1976) and Launder (1989), which include a transport equation for dissipation. Such models 
describe the effects from energy production rates by shear $P=-\overline{u_{i}^{\prime} u_{j}^{\prime}} \partial \bar{u}_{i} / \partial x_{j}$ and buoyancy $B=$ $\beta g \overline{w^{\prime} T^{\prime}}$ on kinetic energy $E$ and dissipation $\varepsilon$. Hence such models can be used to determine the dissipation length-scale $L_{\varepsilon}=E^{3 / 2} / \varepsilon$. For this purpose, we consider the models in the case of local equilibrium, i.e., for small advective and diffusive fluxes and small departures from steady state. Moreover, we assume small departures from the isotropic state so that all corrections in the SOC model in terms of invariants in the departure-from-isotropy tensor (Zeman and Lumley, 1976) can be neglected. Under these conditions the models have the form

$$
\frac{\partial E}{\partial t}=P+B-\varepsilon, \quad \frac{\partial \varepsilon}{\partial t}=\left(\frac{\varepsilon}{E}\right)\left(c_{\varepsilon 1} P+c_{\varepsilon 3} B-c_{\varepsilon 2} \varepsilon\right) .
$$

Zeman and Lumley (1976) used $c_{\varepsilon 1}=0.475, c_{\varepsilon 2}=1.9$, and $c_{\varepsilon 3}=1.9$, while Launder (1989) now prefers $c_{\varepsilon 1}=1, c_{\varepsilon 2}=1.92$, and $c_{\varepsilon 3}=1$. Since

$$
\frac{\partial L_{\varepsilon}}{\partial t}=\frac{3}{2} \frac{E^{1 / 2}}{\varepsilon} \frac{\partial E}{\partial t}-\frac{E^{3 / 2}}{\varepsilon^{2}} \frac{\partial \varepsilon}{\partial t}
$$

the models predict

$$
\frac{\partial L_{\varepsilon}}{\partial t}=\frac{E^{1 / 2}}{\varepsilon}\left\{\left(1.5-c_{\varepsilon 1}\right) P+\left(1.5-c_{\varepsilon 3}\right) B-\left(1.5-c_{\varepsilon 2}\right) \varepsilon\right\}
$$

or, for $\varepsilon \cong P+B$,

$$
\frac{\partial L_{\varepsilon}}{\partial t}=\frac{E^{1 / 2}}{\varepsilon}\left\{\left(c_{\varepsilon 2}-c_{\varepsilon 1}\right) P+\left(c_{\varepsilon 2}-c_{\varepsilon 3}\right) B\right\} .
$$

For positive differences of the coefficients in the above equation (as is the case in Launder (1989)), it predicts that both shear and buoyant convection $(B>0)$ increase the length-scale. This differs from our understanding and numerical results for the flows studied in this paper. This is a simple, perhaps too simplified, analysis, but it shows that SOC models have problems in predicting the dissipation and its related length-scale.

In view of the additional realizability problems of the SOC models (see the Appendix and Schumann (1977)), we now prefer the first-order SGS model, (1) and (2), for LES and propose using $l=\Delta$ (except for the surface adjacent grid cells) for the dissipation and for momentum diffusivity, but the smaller scale, as given in (12), for the SGS eddy diffusivities of vertical diffusion in stably stratified turbulence. This recommendation is consistent with the understanding (Lumley, 1964) that the energy cascade from small to high wave numbers becomes reduced with increasingly stable stratification.

\section{Acknowledgments}

I thank Dr. Chin-Hoh Moeng for providing her LES results (Figure 1(a)) and Dr. Thomas Gerz and Hans Kaltenbach for help in analyzing the results of the direct simulations.

\section{Appendix. The SOC SGS Model}

This appendix summarizes the algebraic SOC model as derived by Schemm and Lipps (1976), Schmidt and Schumann (1989), and Ebert et al. (1989), and identifies its realizability limitations not discussed before. The model corresponds to the "first-order" closure model, as used in this paper, for specific model parameter values, which are given below. The SGS fluxes are approximated in terms of the resolved fields and the SGS kinetic energy $e \equiv \overline{u_{i}^{\prime 2}} / 2$, for which we integrate the closed model-equation

$$
\frac{D e}{D t}=-\overline{u_{i}^{\prime} u_{j}^{\prime}} \frac{\partial \bar{u}_{i}}{\partial x_{j}}+\beta g \overline{w^{\prime} T^{\prime}}+\frac{\partial}{\partial x_{i}}\left[\frac{5}{3} l c_{3 m} e^{1 / 2} \frac{\partial e}{\partial x_{i}}\right]-c_{\mathrm{e} m} \frac{e^{3 / 2}}{l} .
$$

The turbulent heat, mass, and momentum fluxes and their respective anisotropic components,

$$
A_{i j}=\overline{u_{i}^{\prime} u_{j}^{\prime}}-\frac{2}{3} \delta_{i j} e,
$$


are determined from the following set of algebraically approximated SOC equations:

$$
\begin{aligned}
& 0=-\left(1-c_{G m}\right) \frac{2}{3} e\left(\frac{\partial \bar{u}_{i}}{\partial x_{j}}+\frac{\partial \bar{u}_{j}}{\partial x_{i}}\right)+\left(1-c_{B m}\right)\left[\beta g\left(\delta_{i 3} \overline{u_{j}^{\prime} T^{\prime}}+\delta_{j 3} \overline{u_{i}^{\prime} T^{\prime}}-\frac{2}{3} \delta_{i j} \overline{u_{3}^{\prime} T^{\prime}}\right)\right]-c_{R m} \frac{e^{1 / 2}}{l} A_{i j}, \\
& 0=-\left(1-c_{G T}\right) \frac{2}{3} e \frac{\partial \bar{T}}{\partial x_{i}}+\left(1-c_{B T}\right) \beta g \overline{T^{\prime 2}} \delta_{i 3}-c_{R T} \frac{e^{1 / 2}}{l} \overline{u_{i}^{\prime} T^{\prime}}, \\
& 0=-\left(1-c_{G \psi}\right) \frac{2}{3} e \frac{\partial \bar{\psi}}{\partial x_{i}}+\left(1-c_{B \psi}\right) \beta g \overline{\psi^{\prime} T^{\prime}} \delta_{i 3}-c_{R \psi} \frac{e^{1 / 2} \overline{u_{i}^{\prime} \psi^{\prime}}}{l} \\
& 0=-2 \overline{u_{j}^{\prime} T^{\prime}} \frac{\partial \bar{T}}{\partial x_{j}}-c_{\varepsilon T} \frac{e^{1 / 2} \overline{T^{\prime 2}}}{l}, \\
& 0=-\overline{u_{j}^{\prime} \psi^{\prime}} \frac{\partial \bar{T}}{\partial x_{j}}-\overline{u_{j}^{\prime} T^{\prime}} \frac{\partial \bar{\psi}}{\partial x_{j}}-c_{\varepsilon \psi} \frac{e^{1 / 2} \overline{\psi^{\prime} T^{\prime}}}{l} .
\end{aligned}
$$

Here, $\psi$ denotes any passive scalar concentration field. In deriving this set of SOC equations, it has been assumed (Schemm and Lipps, 1976) that local time derivatives, advective fluxes, and anisotropic production rates contribute little to the anisotropic components of the fluxes.

The length-scale $l$ is prescribed as a function of height $z$ above the surface and of the average mesh spacing $\Delta$ by

$$
l=\min \left(\Delta, c_{l} z\right), \quad \Delta=\frac{1}{3}(\Delta x+\Delta y+\Delta z) .
$$

Most model coefficients can be determined from the spectra of kinetic energy and temperature variance in the inertial-convective subrange of locally isotropic turbulence. For details see Schmidt and Schumann (1989) and Ebert et al. (1989).

$$
\begin{gathered}
c_{\varepsilon m}=0.845, \quad c_{\varepsilon T}=c_{\varepsilon \psi}=2.02, \quad c_{G m}=0.55, \quad c_{B m}=0.55, \quad c_{G T}=c_{G \psi}=0.50, \\
c_{B T}=c_{B \Psi}=0.50, \quad c_{R m}=3.50, \quad c_{R T}=c_{R \psi}=1.63, \quad c_{3 m}=0.2, \quad c_{l}=c_{\varepsilon m} .
\end{gathered}
$$

The first-order model is retained if we set $c_{B m}=c_{B T}=c_{B \psi}=1$.

The SOC SGS model, (23)-(26), can be rewritten as

$$
\begin{aligned}
A_{i j} & =-\frac{l}{c_{R m} e^{1 / 2}}\left[\left(1-c_{G m}\right) \frac{2}{3} e\left(\frac{\partial \bar{u}_{i}}{\partial x_{j}}+\frac{\partial \bar{u}_{j}}{\partial x_{i}}\right)+B_{i j}\right], \\
B_{i j} & =\left(1-c_{B m}\right) \beta g\left(\overline{u_{j}^{\prime} T^{\prime}} \delta_{i 3}+\overline{u_{i}^{\prime} T^{\prime}} \delta_{j 3}-\frac{2}{3} \delta_{i j} \overline{u_{3}^{\prime} T^{\prime}}\right), \\
\overline{u_{i}^{\prime} T^{\prime}} & =-\frac{l}{c_{R T} e^{1 / 2}}\left[\left(1-c_{G T}\right) \frac{2}{3} e \frac{\partial \bar{T}}{\partial x_{i}}-\left(1-c_{B T}\right) \beta g \overline{T^{\prime 2}} \delta_{i 3}\right], \\
\overline{u_{i}^{\prime} \psi^{\prime}} & =-\frac{l}{c_{R T} e^{1 / 2}}\left[\left(1-c_{G \psi}\right) \frac{2}{3} e \frac{\partial \bar{\psi}}{\partial x_{i}}-\left(1-c_{B \psi}\right) \beta g \overline{T^{\prime} \psi^{\prime}} \delta_{i 3}\right], \\
\overline{T^{\prime 2}} & =-\frac{2 l}{c_{\varepsilon T} e^{1 / 2}}\left[\overline{u_{i}^{\prime} T^{\prime}} \frac{\partial T}{\partial x_{i}}\right] .
\end{aligned}
$$

In this form it becomes obvious that the buoyancy fluxes cause a deviation from the pure gradientflux form.

The fluxes and variances can be determined explicitly by solving the above linear system of equations. Using the abbreviations

$$
\begin{gathered}
c_{v}=\frac{2}{3} \frac{1-c_{G m}}{c_{R m}}, \quad c_{B}=\frac{4}{9} \frac{\left(1-c_{B m}\right)\left(1-c_{G T}\right)}{c_{R m} c_{R T}}, \\
c_{\gamma}=\frac{2}{3} \frac{1-c_{G T}}{c_{R T}}, \quad c_{\psi}=\frac{2}{3} \frac{1-c_{G \psi}}{c_{R \psi}}
\end{gathered}
$$

and

$$
G=\frac{2\left(1-c_{B T}\right)}{c_{R T} c_{\varepsilon T}} \frac{\beta g l^{2}}{e}, \quad G_{\psi}=\frac{1-c_{B \psi}}{c_{R \psi} c_{\varepsilon \psi}} \frac{\beta g l^{2}}{e}
$$


we obtain

$$
\begin{aligned}
A_{11} & =-c_{v} l e^{1 / 2} 2 \frac{\partial \bar{u}}{\partial x}-c_{B} l^{2} \beta g\left\{\frac{\partial \bar{T}}{\partial z}-\frac{G\left(\partial \bar{T} / \partial x_{i}\right)^{2}}{1+G \partial \bar{T} / \partial z}\right\}, \\
A_{22} & =-c_{v} l e^{1 / 2} 2 \frac{\partial \bar{v}}{\partial y}-c_{B} l^{2} \beta g\left\{\frac{\partial \bar{T}}{\partial z}-\frac{G\left(\partial \bar{T} / \partial x_{i}\right)^{2}}{1+G \partial \bar{T} / \partial z}\right\}, \\
A_{33} & =-c_{v} l e^{1 / 2} 2 \frac{\partial \bar{w}}{\partial z}+2 c_{B} l^{2} \beta g\left\{\frac{\partial \bar{T}}{\partial z}-\frac{G\left(\partial \bar{T} / \partial x_{i}\right)^{2}}{1+G \partial \bar{T} / \partial z}\right\}, \\
\overline{w^{\prime} T^{\prime}} & =-c_{\gamma} l e^{1 / 2}\left[\frac{\partial \bar{T}}{\partial z}-\frac{G\left(\partial \bar{T} / \partial x_{i}\right)^{2}}{1+G \partial \bar{T} / \partial z}\right], \\
\overline{T^{\prime 2}} & =2 \frac{c_{\gamma}}{c_{\varepsilon T}} \frac{l^{2}}{1+G \partial \bar{T} / \partial z}\left(\frac{\partial \bar{T}}{\partial x_{i}}\right)^{2}, \\
\overline{\psi^{\prime} T^{\prime}} & =\frac{1}{c_{\varepsilon \psi}} \frac{1}{1+G_{\psi} \partial \bar{T} / \partial z}\left[\left(c_{\psi}+c_{\gamma}\right) l^{2} \frac{\partial \bar{\psi}}{\partial x_{j}} \frac{\partial \bar{T}}{\partial x_{j}}-\frac{c_{\gamma} G l^{2}}{1+G \partial \bar{T} / \partial z} \frac{\partial \bar{\psi}}{\partial z}\left(\frac{\partial \bar{T}}{\partial x_{i}}\right)^{2}\right] .
\end{aligned}
$$

Once these fluxes are computed, the remaining flux components follow from (30) to (33). In order to ensure finite positive solutions, $1+G \partial \bar{T} / \partial z$ and $1+G_{\psi} \partial \bar{T} / \partial z$ have to be replaced by unity if they become less than one, respectively.

One purpose of this appendix is to point out a further realizability problem related to this SOC SGS model. This problem was noted by Krettenauer (1991) in applications of the present model in simulations of the CBL using a very coarse grid. He showed that the model predicts negative shear-induced production rates $P$ near the surface and explained this fact as follows: The production rate is $P=-\frac{1}{2} \overline{u_{i}^{\prime} u_{j}^{\prime}} D_{i j}$, where $D_{i j}=\partial \bar{u}_{i} / \partial x_{j}+\partial \bar{u}_{j} / \partial x_{i}$ is the resolved velocity deformation tensor. Using (30) and the continuity equation, $D_{i i}=0$, results in

$$
P=\frac{1}{2} K_{m}\left(D_{i j}\right)^{2}+\frac{l}{2 c_{R m} e^{1 / 2}} B_{i j} D_{i j}
$$

Here, the first part, with $K_{m}=c_{v} l e^{1 / 2}$, is positive definite, but the second term can be of either sign. If we insert the explicit equations for the fluxes, we obtain

$$
P=\frac{1}{2} K_{m}\left(D_{i j}\right)^{2}+\frac{3}{2} c_{B} \beta g l^{2}\left\{D_{13} \frac{\partial \bar{T}}{\partial x}+D_{23} \frac{\partial \bar{T}}{\partial y}+D_{33}\left(\frac{\partial \bar{T}}{\partial z}-\frac{G\left(\partial \bar{T} / \partial x_{i}\right)^{2}}{1+G \partial \bar{T} / \partial z}\right)\right\}
$$

Obviously the last term may balance the first term and cause a negative shear production rate of large magnitude (even exceeding the buoyant production rate). This can happen in particular for coarse grids, i.e., for large values of $l^{2}$. In practice, this causes problems mainly in the lowest grid cells where both the temperature and the velocity derivatives may become large. In numerical applications, the problem is overcome by replacing negative shear production rates by zero. The realizability problem is absent from the first-order closure model and for this reason we now prefer the simpler approach. However, as can be seen from the comparisons in Figure 3, the quantitative consequences of this problem are small.

\section{References}

Bougeault, P., and Lacarrère, P. (1989). Parameterization of orography-induced turbulence in a mesobeta-scale model. Monthly Weather Rev., 117, 1872-1890.

Brost, R.A., and Wyngaard, J.C. (1978). A model study of the stably stratified planetary boundary layer. J. Atmos. Sci., 35, $1427-1440$.

Deardorf, J.W. (1973). The use of subgrid transport equations in a three-dimensional model of atmospheric turbulence. $J$. Fluids Engrg., 95, 429-438.

Deardorff, J.W. (1980). Stratocumulus-capped mixed layers derived from a three-dimensional model. Boundary-Layer Meteorol., $18,495-527$.

Druilhet, A, Frangi, J.P., Guedalia, D., and Fontan, J. (1983). Experimental studies of the turbulence structure parameters of the convective boundary layer. J. Climatol. Appl. Meteorol., 22, 594-608. 
Ebert, E.E., Schumann, U., and Stull, R.B. (1989). Nonlocal turbulent mixing in the convective boundary layer evaluated from large-eddy simulation. J. Atmos. Sci., 46, 2178-2207.

Gerz, T., and Schumann, U. (1991). Direct simulation of homogeneous turbulence and gravity waves in sheared and unsheared stratified flows. In Turbulent Shear Flows, Vol. 7 (F. Durst et al., eds.), pp. 27-45. Springer-Verlag, Berlin.

Gerz, T., Schumann, U., and Elghobashi, S.E. (1989). Direct numerical simulation of stratified homogeneous turbulent shear flows. J. Fluid Mech., 200, 563-594.

Guillemet, B., Isaka, H., and Mascart, P. (1983). Molecular dissipation of turbulent fluctuations in the convective mixed layer. I: Height variations of dissipation rates. Boundary-Layer Meteorol., 27, 141-162.

Hunt, J.C.R., Kaimal, J.C., and Gaynor, J.E. (1985). Some observations of turbulence structure in stable layers. Quart. J. Roy. Meteorol. Soc., 111, 793-815.

Hunt, J.C.R., Stretch, D.D., and Britter, R.E. (1988). Length scales in stably stratified turbulent flows and their use in turbulence models. In Stably Stratified Flows and Dense Gas Dispersion (J.S. Puttock, ed.), pp. 285-321. Clarendon Press, Oxford.

Hunt, J.C.R., Moin, P., Moser, R.D., Spalart, P., Mansour, N.N., Kaimal, J.C., and Gaynor, E. (1989). Cross correlation and length scales in turbulent flows near surface. In Advances in Turbulence, Vol. 2 (H.-H. Fernholz and H.E. Fiedler, ed.), pp. 128-134. Springer-Verlag, Berlin.

Isaka, H., and Guillemet, B. (1983). Molecular dissipation of turbulent fluctuations in the convective mixed layer. II: Height variations of characteristic time scales and experimental test of molecular dissipation models. Boundary-Layer Meteorol., 27, 257-279.

Krettenauer, K. (1991). Numerische Simulation turbulenter Konvektion über gewellten Flächen. Dissertation, Report DLR-FB91-12, DLR Oberpfaffenhofen.

Launder, B.E. (1975). On the effects of a gravitational field on the turbulent transport of heat and momentum. J. Fluid Mech., 67, 569-581.

Launder, B.E. (1989). The prediction of force field effects on turbulent shear flows via second-moment closure. In Advances in Turbulence, vol. 2 (H.-H. Fernholz, and H.E. Fiedler, eds.), pp. 338-358. Springer-Verlag, Berlin.

Lilly, D.K. (1967). The representation of small-scale turbulence in numerical simulation experiments. In Proc. IBM Sci. Comput. Symp. on Environmental Science, pp. 195-210. Thomas J. Watson Research Center, Yorktown Heights, N.Y., 14-16 November 1966. IBM Form 320-1951.

Lilly, D.K., Waco, D.E., and Adelfang, S.I. (1974). Stratospheric mixing estimated from high-altitude turbulence measurements. J. Appl. Meteorol, 13, 488-493.

Lumley, J.L. (1964). The spectrum of nearly inertial turbulence in a stably stratified fluid. J. Atmos. Sci., 21, 99-102.

Mason, P.J. (1989). Large eddy simulation of the convective atmospheric boundary layer. J. Atmos. Sci., 46, 1492-1516.

Mason, P.J., and Derbyshire, S.H. (1990). Large-eddy simulation of the stably-stratified atmospheric boundary layer. BoundaryLayer Meteorol., 53, 117-162.

Mellor, G.L., and Yamada, T. (1982). Development of a turbulence closure model for geophysical fluid problems. Rev. Geophys. Space Phys., 20, 851-875.

Moeng, C.-H., and Wyngaard, J.C. (1989). Evaluation of turbulent transport and dissipation closures in second-order modeling. J. Atmos. Sci., 46, 2311-2330.

Nieuwstadt, F.T.M. (1990). Direct and large-eddy simulation of free convection. In Proc. 9th Internat. Heat Transfer Conf., Vol. I, pp. 37-47. Jerusalem, 19-24 August 1990.

Ozmidov, R.V. (1965). On the turbulent exchange in a stably stratified ocean. Bull. Acad. Sci. U.S.S.R. Atmos. Ocean. Phys., 1, 493-497.

Schemm, C.E., and Lipps, F.B. (1976). Some results of a simplified three-dimensional numerical model of atmospheric turbulence. J. Atmos. Sci., 33, 1021-1041.

Schmidt, H., and Schumann, U. (1989). Coherent structure of the convective boundary layer derived from large-eddy simulations. J. Fluid Mech., 200, 511-562.

Schumann, U. (1977). Realizability of Reynolds stress turbulence models. Phys. Fluids, 20, 721-725.

Schumann, U. (1990). Large-eddy simulation of the upslope boundary layer. Quart. J. Roy Meteorol. Soc., 116, 637-670.

Thorpe, S.A. (1978). On the shape and breaking of finite amplitude internal gravity waves in a shear flow. J. Fluid Mech., 85, 7-31.

Vinnichenko, N.K., Pinus, N.Z., Shmeter, S.M., and Shur, G.N. (1973). Turbulence in the Free Atmosphere, pp. 120-127. Consultants Bureau, New York.

Webster, C.A.G. (1964). An experimental study of turbulence in a density stratified shear flow. J. Fluid Mech., 19, $221-245$.

Weinstock, J. (1978). Vertical diffusion in a stably stratified fluid. J. Atmos. Sci., 35, 1022-1027.

Weinstock, J. (1990). Saturated and unsaturated spectra of gravity waves and scale-dependent diffusion. J. Atmos. Sci., 47, 2211-2225.

Zeman, O., and Lumley, J.L. (1976). Modeling buoyancy driven mixed layers. J. Atmos. Sci., 33, 1974-1988. 